\title{
The experimental study of elastic-hysteresis properties of rubber elements of sleeve-pin couplings
}

\author{
Dmitry Nasonov ${ }^{1}$, Vladimir Ilichev ${ }^{2}$, Vladimir Raevsky ${ }^{3}$ \\ ${ }^{1}$ Department of Machines' Vibroacoustics, Mechanical Engineering Research Institute of the Russian \\ Academy of Sciences, Moscow, Russian Federation \\ ${ }^{2}$ Department of Heat Engines and Hydraulic Machines, Bauman State Technical University (Kaluga \\ Branch), Kaluga, Russian Federation \\ ${ }^{3}$ Department of Informatics and Information Technologies, Kaluga State University named after K. E. \\ Tsiolkovski, Kaluga, Russian Federation \\ ${ }^{1}$ Corresponding author \\ E-mail: ${ }^{1}$ nasonovda@yandex.ru, ${ }^{2}$ patrol8@yandex.ru, ${ }^{3}$ var-77@mail.ru
}

Received 13 May 2021; received in revised form 28 May 2021; accepted 4 June 2021

DOI https://doi.org/10.21595/vp.2021.22055

Check for updates

Copyright $(02021$ Dmitry Nasonov, et al. This is an open access article distributed under the Creative Commons Attribution License, which permits unrestricted use, distribution, and reproduction in any medium, provided the original work is properly cited.

\begin{abstract}
The work is devoted to the study of the ability of rubber sleeves of different configuration to dampen the vibration energy. The processing of the experiment results showed a significant difference in the elastic hysteresis properties of different rubber element designs and made it possible to choose an element with preferable vibration-isolating properties for use in drive units. Recommendations for further improvement of the design and provision of certain properties of rubber elastic elements are offered.
\end{abstract}

Keywords: rubber elastic element, sleeve and pin coupling, elastic hysteresis, vibro-isolator, shock absorber.

\section{Introduction}

Sleeve - pin couplings are widely used in rotary machines to connect the drive shafts and the driven machine. Electric motors, turbines, diesel engines are used as the shaft and pumps, fans, compressors, blowers and other rotary units as the driven machine. Also, this type of couplings is very often used in transport installations.

Sleeve - pin couplings allow relative axial and radial shaft displacements, which are necessary during thermal expansions, in cases where the shaft mechanism does not allow rotor-to-stator displacements, for example, when axial forces are grasped by a thrust bearing, to compensate shaft misalignments and to reduce shaft mutual interference in rotor systems.

The main elements of sleeve - pin couplings, providing the functions of vibro-isolation, cushioning and compensation of misalignment of connected shafts, are rubber elastic elements [1] - bushings fixed on metal pins. The advantage of these coupling elements is that they can be easily replaced with similar ones in case of wear or other types of damage.

The properties of rubber sleeves can be changed by selecting the grade and composition of the rubber from which they are made. The most important properties in this case are the stiffness and damping ability of the rubber mass [2].

If the rubber stiffness increases, it can carry more loads, but the compensating properties of the coupling deteriorate - at misalignment of the rotating shafts connected, they will be more deformed, therefore, there will be increased vibrations of the rotor system. The article [3] is devoted to measuring the stiffness characteristics of rubber elements of sleeve-pin couplings.

The vibration level of the rotor system is greatly influenced by the damping (shock absorbing) capacity of rubber couplings, i.e. the ability to dampen vibrations by dissipating the vibration energy in the rubber mass during cyclic loading. The damping capacity of rubber is determined by the area of the closed part of the graph of its deformation when the load is applied and when it is unloaded - the so-called hysteresis loop.

Physically, the hysteresis phenomenon is explained by the fact that only the part of the 
mechanical energy spent on deformation is returned during unloading. The loss of mechanical energy of deformation is explained by its irreversible dissipation in the form of heat during the processes of internal structural friction (interaction of molecules) in the material. If the elastic sample under study is subjected to numerous repeated loading-unloading cycles, the level of mechanical energy loss becomes constant for each cycle (a stable hysteresis loop is established). This happens due to the stabilization of changes in the structure of the elastic sample [4].

The problem under consideration is relevant because the use of rubber elastic elements often does not take into account the hysteresis of elastic properties, which reduces the quality of machine design.

\section{Tasks and methods of their solution}

The aim of the work was to develop experimentally justified methodology for selecting the rubber sleeve design that provides the best vibration-absorbing and shock-absorbing properties with the same overall dimensions and material of the sleeves. Studies on this problem have not yet received consecration in the scientific literature, but are very relevant for improving the designed rotor installations.

Let's consider the theoretical prerequisites for improving the above properties of rubber sleeves.

To strengthen the vibro-isolating and shock-absorbing properties of the elastic elements of couplings, it is necessary to achieve an increase in their vibration energy losses, which can be determined by the curve of loading-unloading C1B2C (Fig. 1), which is a dependence of the deformation on the applied load.

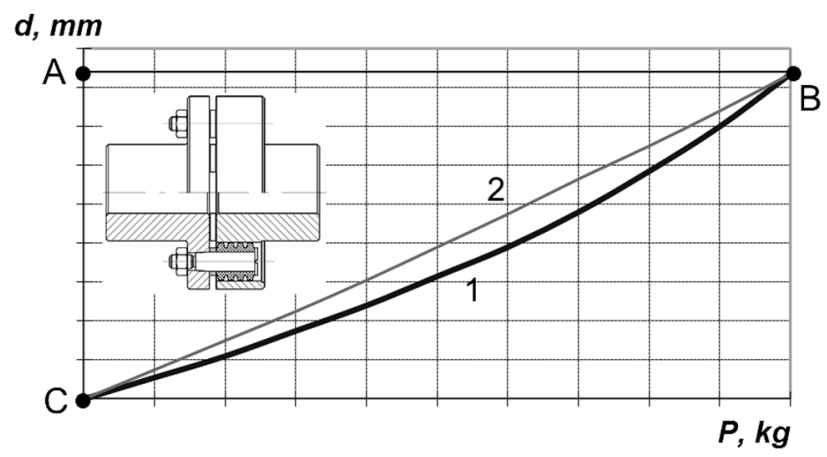

Fig. 1. Hysteresis loop of an elastic sample

The curve allows you to estimate the energy expended and energy returned, as well as hysteresis losses. When the rubber is loaded, mechanical work proportional to the area AB1CA is expended; when it is unloaded, work proportional to the area $\mathrm{AB} 2 \mathrm{CA}$ is returned. The area of the hysteresis loop $\mathrm{C} 1 \mathrm{~B} 2 \mathrm{C}$ is proportional to the hysteresis loss. The vibro-absorbing properties of a rubber sample can be numerically evaluated with the help of the coefficient of useful elasticity the ratio of returned work to expended work (\%).

The hysteresis properties of rubber elastic elements depend on their design, rubber composition and manufacturing technology (which determine the internal material structure) [5] and can be determined experimentally for specific sleeve designs.

The methodology developed by the authors for the experimental determination of the properties of rubber elastic elements consists of a number of processes.

1) The device was designed and manufactured that simulates the way of fixing the sleeves on the metal pins in the coupling and sets the direction of the load application $P$.

2) A suitable device for application of adjustable load to the sleeve - press "Universal ripping machine ZIM R-10" - has been selected. 
3) To control the applied force, a compression dynamometer has been installed between the press loading device and the fixture element that transmits the load to the tested elastic element.

4) In order to measure the deformation of the rubber mass, sensitive displacement indicators are attached to the part of the fixture that directly transmits the load to the sleeve and simultaneously deforms it. Since the static characteristics of the sleeves were measured, the readings were recorded only after the unchanged values had been established at the indicators.

5) Measurements were made and their deformation dependences on the applied force have been represented for rubber elements of sleeve-pin couplings of different shapes.

6) The obtained graphs have been used to calculate the areas of hysteresis loops which are proportional to the work of deformation converted into thermal energy.

7) Based on the found work, the vibration-damping properties of different rubber sleeve configurations have been compared and recommendations for their improvement have been worked out.

The design of the experimental setup is shown in Fig. 2. The designs of the tested sleeves are presented in Fig. 3.

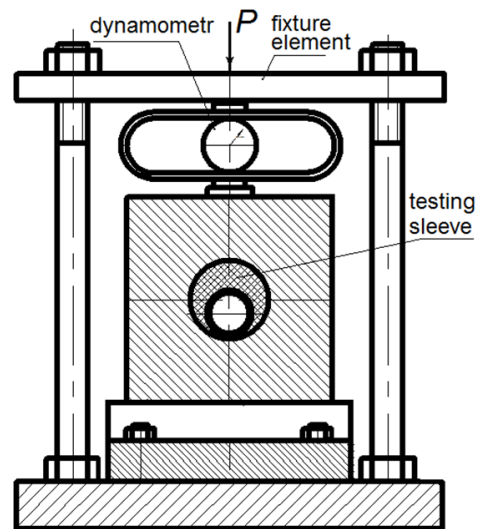

Fig. 2. Design of the experimental setup

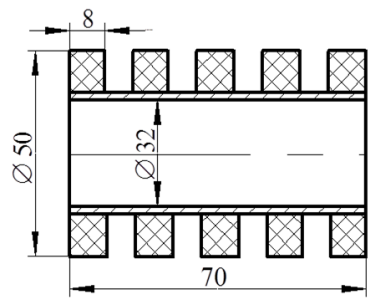

a) Symmetric sleeve

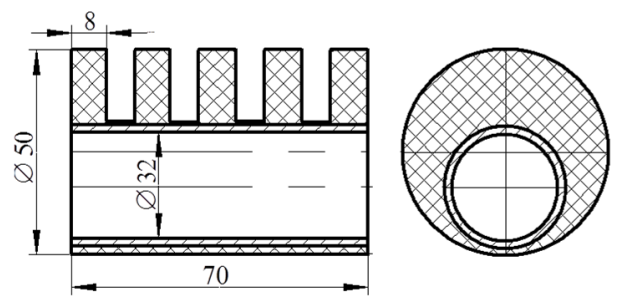

b) Asymmetric sleeve

Fig. 3. Designs of the tested rubber sleeves

The rubber mass of the elastic elements is secured by vulcanization to a metal sleeve, which is slipped onto the coupling pin. To reduce the stiffness of the rubber part of the elastic elements, annular bands have been made.

The first sleeve design is symmetrical (Fig. 3(a)), which is traditionally used in most sleeve-pin couplings. The second modernized sleeve design proposed by the authors is asymmetrical (Fig. 3(b), with the greatest thickness of the rubber mass in the direction of deformation, and with the least thickness on the unloaded side. The asymmetrical design of the rubber bushings allows the design and dimensions of all other elements of the coupling to be fully preserved, i.e. to modernize it in the easiest and least expensive way. The conducted researches have shown the advantages of asymmetrical sleeve design in terms of better vibration damping. 


\section{Results}

Thus, hysteresis characteristics of the bushings tested were determined experimentally. The results of measurements are shown in Table 1 and in Fig. 4, in which the dependences of the deformation of the rubber sleeves on the value of the applied force are drawn.

Table 1. Test results of rubber sleeves

\begin{tabular}{|c|c|c|c|c|}
\hline \multirow{2}{*}{$\begin{array}{c}\text { Loading } \\
P, \mathrm{~kg}\end{array}$} & \multicolumn{4}{|c|}{ Deformation of the sleeve $d, \mathrm{~mm}$} \\
\cline { 2 - 5 } & Symmetric sleeve & \multicolumn{2}{c|}{ Asymmetric sleeve } \\
\cline { 2 - 5 } & straightforward stroke & reverse course & straightforward stroke & reverse course \\
\hline 20 & 0 & 0,03 & 0 & 0,05 \\
\hline 40 & 0,11 & 0,165 & 0,15 & 0,27 \\
\hline 80 & 0,24 & 0,29 & 0,4 & 0,59 \\
\hline 120 & 0,34 & 0,375 & 0,63 & 0,85 \\
\hline 160 & 0,41 & 0,45 & 0,84 & 1,07 \\
\hline 200 & 0,46 & 0,5 & 1,05 & 1,25 \\
\hline 240 & 0,51 & 0,55 & 1,22 & 1,42 \\
\hline 280 & 0,55 & 0,58 & 1,4 & 1,56 \\
\hline 320 & 0,59 & 0,61 & 1,55 & 1,68 \\
\hline 360 & 0,625 & 0,63 & 1,71 & 1,83 \\
\hline 400 & 0,65 & 0,65 & 1,83 & \\
\hline
\end{tabular}

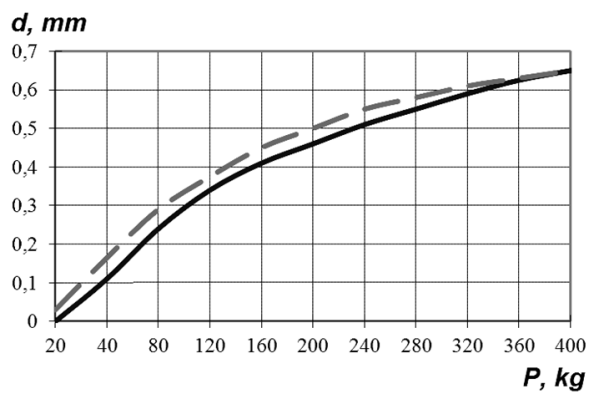

a) Symmetric sleeve

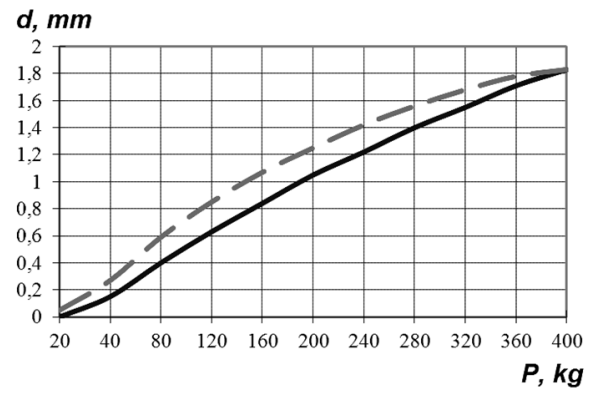

b) Asymmetric sleeve

Fig. 4. Results of measuring the deformation of rubber sleeves

\section{Discussion}

Due to the occurrence of internal friction in the rubber mass, the deformation graphs during loading and unloading (during the implementation of the forward and reverse stroke of the press) do not coincide, forming hysteresis loops (Fig. 4).

The useful elasticity coefficients calculated from the graphs are:

- for the symmetric sleeve $86.3 \%$;

- for the asymmetric sleeve $82.0 \%$.

Thus, a higher percentage of mechanical energy is lost in the load-discharge cycles of a asymmetric sleeve. This can be explained by the greater thickness of the rubber mass in the direction of the greatest amplitude of cyclic load. This direction in the sleeve-pin coupling is circumferential, with the torque load vector directed tangentially to the center diameter of the coupling pin centers. The sleeves in the coupling must be positioned so that the load vector is in the direction of the rubber mass of the greatest thickness.

In the case under consideration, the design of sleeve-pin couplings a significant dampening of alternating forces and moments arising from shaft misalignment can be achieved by selecting the required elastic and hysteresis properties of rubber elements. In this case, it is necessary to reduce the coefficient of useful elasticity by increasing the thickness of the rubber mass in the direction 
of the maximum cyclic load and using other known methods of increasing the hysteresis loop area of elastic materials (use of crystallizing rubbers, white soot impurities and other fillers in the composition of the rubber [6]).

\section{Conclusions}

The novelty of the work consists in the fact that it developed and verified the methodology of experimental determination of elastic-hysteresis properties of rubber elastic elements of bushing and finger couplings, which was not used before.

The experimental data confirmed the presence of elastic static hysteresis of the sleeves due to the presence of internal molecular friction in the structure of the rubber mass.

The research methodology can be used to study the properties of any designs of elastic inserts made from different damping materials and used not only in couplings, but also in vibration isolators, compensators and shock absorbers. It allows you to select the necessary damping properties of these elastic elements.

However, it should be taken into account that in some elements of structures elastic-hysteresis energy losses may be undesirable, because they reduce the efficiency of the mechanical system.

\section{References}

[1] Polukoshko Svetlana, Martinovs Andris, Zaicevs Edgars Influence of rubber ageing on damping capacity of rubber vibration absorber. Vibroengineering Procedia, Vol. 19, 2018, p. 103-109.

[2] Lu Guang Qing, Xu Kun Peng, Peng Wen Bin, Ragulskis Minvydas, Wang Bo Coupling dynamic stiffness identification of mechanical assembly with linear connection by the second indirect scheme of inverse substructuring analysis. Vibroengineering Procedia, Vol. 20, 2018, p. 24-29.

[3] Ilyichev V. Y., Nasonov D. A. Experimentally - analytical study and correction of static and dynamic characteristics of rubber-finger couplings, Izvestia of Tula State University. Technical Sciences, Vol. 3, 2011, p. 46-52.

[4] Stankiewicz Marek, Targosz Jan Research methodology for vibration isolation materials. Vibroengineering Procedia, Vol. 6, 2015, p. 30-35.

[5] Zhang Yang, Chen Zhaobo, Jiao Yinghou, Wang Xiaoyu Hysteresis linearization control of a novel hybrid vibration isolator. Journal of Vibroengineering, Vol. 19, Issue 3, 2017, p. 1557-1568.

[6] King Yang H. Basic Finite Element Method as Applied to Injury Biomechanics. Academic Press, 2018, p. 231-256. 